\title{
On Lifetimes of Dusty Debris Disks around A-type Stars
}

\author{
Ramiro de la Reza ${ }^{1}$ and Carolina Chavero ${ }^{2}$ \\ ${ }^{1}$ Observatório Nacional, Ro de Janeiro, Brazil \\ email: delareza@on.br \\ ${ }^{2}$ Observatorio Astronómico de Córdoba, Córdoba, Argentina \\ email: carolina@oac.uncor.edu
}

\begin{abstract}
Using a collection of 40 debris disks (DD) around dwarf A-type stars (from which 17 are spatially resolved) having well determined stellar ages (Vican 2012), we found that for a collisional self-stirring model of flux distributions (Kenyon \& Bromley, 2008, 2010) dusty DD occupy for a large part the terminal age positions corresponding the end of the MS phase. This situation does not necessarily mean that the dust generation in these DD is finished at this stage. Dusty DD can continue their lives in the next Post-MS stages, as is the case of the recent first resolved dusty DD with planets around the K-type subgiant star $\kappa \mathrm{CrB}$. This star is considered a "retired" A star (Bonsor et al. 2013). In order to explain the apparent lack of dusty DD in the giant phase, we propose here that this could be due, in part, to a dilution of any planetesimal belt existing around these stars, with the corresponding diminishing of collisions and subsequent dust generation. This dilution is provoked by the dynamical expansion of this belt produced by the important stellar mass loss during the RGB phase. This scenario is being studied by means of numerical simulations by de la Reza, Roig \& Chavero 2013 (in preparation).
\end{abstract}

Keywords. planetary formation, debris disk, circumstellar disk

\section{Motivation}

The recent detection and spatial resolving of the first dusty debris disk (DD) with planets around the K-type subgiant star $\kappa \mathrm{CrB}$ (Bonsor et al. 2013) and the apparent absence of signatures of DD in giant stars, lead us to study the probable end of dust generation in DD. Early studies on evolution of DD have suffered from the lack of knowledge of well determined stellar ages. To study the end of dust production in DD stars around A-type stars, we use a recent published list of homogeneous age data for A-type DD stars, specially selected for studies with Herschel (Vican 2012). We also use the most complete collection of resolved (17) and non-resolved (23) A-type DD, totaling 40 objects which have been measured with Spitzer and Hershel observatories.

\section{The collisional disks model}

We consider the self-stirring collisional numerical models of DD and planet formation of Kenyon \& Bromley $(2008,2010)$ hereafter (KB08, KB10). The emission fluxes of the new formed dust resulting from collisions are calculated (for different dimensions, strengths of the initial planetesimals, stellar masses and for Spitzer and Herschel selected wavelengths). Here, we use fluxes at $24 \mu \mathrm{m}$ and $70 \mu \mathrm{m}$ for which we dispose the most complete data. We also consider that radiation at $70 \mu \mathrm{m}$ covers an important part of the disk (Sibthorpe et al. 2010, Booth et al. 2013). In the figures the fluxes ratios at $70 \mu \mathrm{m}$ over $24 \mu \mathrm{m}$ are presented in the form: $[70-24]=\log \left(24 \mathrm{~F}_{70}\right)-\log \left(70 \mathrm{~F}_{24}\right)$, (Chavero 

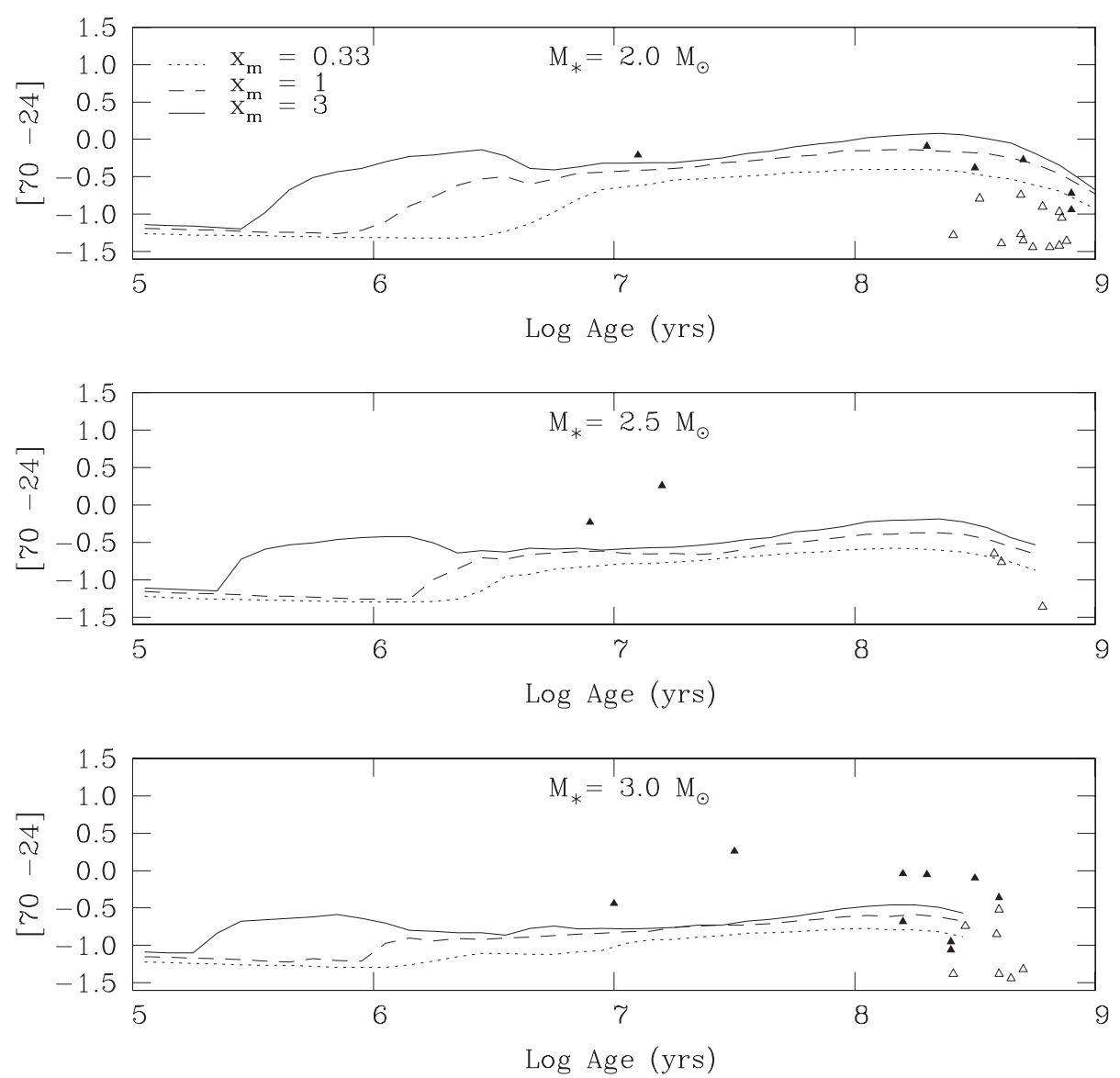

Figure 1. In this figure are superposed the observed flux ratios (in the form of $[70-24]=$ $\left.\log \left(24 \mathrm{~F}_{70}\right)-\log \left(70 \mathrm{~F}_{24}\right)\right)$ of the $40 \mathrm{DD}$ stars at $70 \mu \mathrm{m}$ and $24 \mu \mathrm{m}$ on the distribution of dust radiation of the collisional model of Kenyon \& Bromley (2008) in function of age. In this model the initial planetesimals are small with a radius of $\leqslant 1 \mathrm{~km}$. Three stellar masses of $2.0 \mathrm{M} \odot$, $2.5 \mathrm{M} \odot$, and $3.0 \mathrm{M} \odot$ are considered representing the different A-type stars used here. Xm are model parameters with values from 3.0, 1.0 and 0.33 corresponding respectively to disks with smaller masses. Filled points correspond to resolved disks whereas open symbols to unresolved disks.

2009), where the fluxes are those calculated in KB08 for stellar masses of 2.0, 2.5 and $3.0 \mathrm{M} \odot$, masses that we consider representative for all types of A stars.

\section{References}

Bonsor, A. \& Wyatt, M. C. 2010, MNRAS, 409, 163

Bonsor, A., kennedy, G. M., et al. 2013, MNRAS, 431, 4, 3025

Booth, M., et al. 2013, MNRAS, 248, 1263

Chavero, C. 2009, PhD thesis at Observatorio Nacional, Rio de Janeiro, Brazil

Kenyon, S. J. \& Bromley, B. C. 2008, ApJS 179, 451

Kenyon, S. J. \& Bromley, B. C. 2010, ApJS, 188, 242

Sibthorpe, B., \& et al. 2010, A\&A, 518.130

Vican, L. 2012, $A J$ 143, 135 